\title{
Characterization of the DuPont photopolymer for three-dimensional holographic storage
}

\author{
Kevin Curtis and Demetri Psaltis
}

\begin{abstract}
DuPont's HRF-150 photopolymer film is investigated for use in three-dimensional holographic memories. Measurements of sensitivity, hologram persistence, the lateral spread of the photoinitiated reaction, and the variation of diffraction efficiency with modulation depth, spatial frequency and tilt angle, and intensity are reported. We observed that the diffraction efficiency of the HRF-150 photopolymer for a given exposure decreases with increases in intensity and grating tilt angle. The holograms were nondestructively reconstructed for long periods of time at room temperature. The photoinitiated reaction spread less than $100 \mu \mathrm{m}$ over a period of $16 \mathrm{~h}$.
\end{abstract}

Key words: Holography, holographic memories, photopolymers.

Three-dimensional holographic disk systems can be used as memories and correlation devices with high storage-density and correlation rates. ${ }^{1,2}$ DuPont's photopolymer HRF-150 has excellent sensitivity and good resolution for transmission holograms recorded with blue-green light. ${ }^{3}$ Thus it is a likely candidate for use as a holographic storage material. In addition, angular multiplexing of images in standard films ${ }^{4}$ and thick films from solutions either cast or spin coated onto glass substrates ${ }^{5}$ has been demonstrated. Several recording parameters for single holograms have been previously investigated.6,7 This paper presents results on sensitivity, hologram persistence, the lateral spread of the photoinitiated reaction, and the variation of the diffraction efficiency with modulation depth, spatial frequency and tilt angle, and intensity. These are all important characteristics for evaluation of these photopolymer films as media for holographic storage applications.

HRF-150 photopolymer films consists of $38-\mu \mathrm{m}$ thick photopolymer film sandwiched between two thin sheets of Mylar. Hologram storage was performed with the setup shown in Fig. 1. The object beam is incident normal to the surface, while the

When this research was performed, the authors were with the Department of Electrical Engineering, California Institute of Technology, MS 116-81, Pasadena, California 91125. K. Curtis is now with the AT\&T Bell Laboratories, Murray Hill, New Jersey 07974.

Received 23 November 1993; revised manuscript received 7 February 1994.

0003-6935/94/235396-04\$06.00/0.

(1) 1994 Optical Society of America. reference beam is incident at an angle $\theta$. Both beams are plane waves at $488 \mathrm{~nm}$, and the film is mounted on a glass substrate. After recording, the hologram is fixed by exposure of the film to uniform UV light for $45 \mathrm{~s}$. We define diffraction efficiency as diffracted intensity divided by incident intensity after the reference beam is re-Bragg matched to adjust for film shrinkage. ${ }^{7}$

The sensitivity curve of the material is shown in Fig. 2, in which the diffraction efficiency of the first diffracted order is plotted versus the total exposure energy. The intensity of each beam was kept constant at $2 \mathrm{~mW} / \mathrm{cm}^{2}$ with $\theta \approx 18^{\circ}$, and the exposure time was varied to obtain the data in Fig. 2. When the diffraction efficiency exceeded $20 \%$, a considerable amount of energy was diffracted into the second and the neagtive-first orders. Notice that the saturation exposure is $\sim 80 \mathrm{~mJ} / \mathrm{cm}^{2}$. Similar measurements were reported in Refs. 4 and 7. A saturation exposure of $\sim 300 \mathrm{~mJ} / \mathrm{cm}^{2}$ was reported in Ref. 7 , which is significantly different from what is reported here, most likely because the recording wavelength was $514 \mathrm{~nm}$. Thus the film appears to be more sensitive at $488 \mathrm{~nm}$.

We also measured the effect of the intensity on the photopolymer's sensitivity. Keeping the intensities of the two beams equal, $\theta \sim 20^{\circ}$, and the total exposure of $20 \mathrm{~mJ} / \mathrm{cm}^{2}$, we measured the diffraction efficiency as we changed the total intensity of the two beams. Figure 3 shows that as the intensity is increased, the film becomes less effective. Since the photoinitiated reaction is a free-radical reaction, it can be slowed or stopped by inhibitors. Free radicals themselves are efficient inhibitors; therefore increas- 


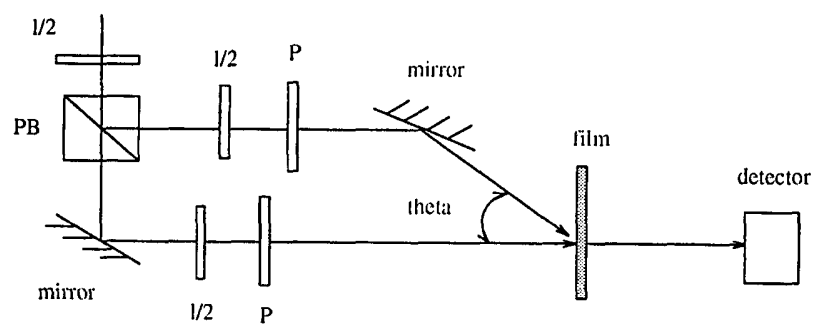

Fig. 1. Recording geometry used for measurements with the following elements: P's polarizers; $1 / 2$ 's, half-wave plates; $\mathrm{PB}$, polarizing beam splitter.

ing intensity, which generates more free radicals, can inhibit the reaction.

The modulation depth is defined as $m=$ $2 R S /\left(R^{2}+S^{2}\right)$, where $R$ and $S$ are the amplitudes of the reference and the signal beams. Figure 4 shows the diffraction efficiency versus $m$ for $\theta \sim 20^{\circ}$ with the total exposure kept constant at $20 \mathrm{~mJ} / \mathrm{cm}^{2}$. The total intensity varied from $4 \mathrm{~mW} / \mathrm{cm}^{2}$ to $\sim 2 \mathrm{~mW} / \mathrm{cm}^{2}$. The curve shows that if $m<0.2$, the diffraction efficiency is small. This is probably because at low modulation the large background intensity polymerizes the material uniformly, which impedes monomer diffusion. Measurements of the dependence of the efficiency on the modulation depth were also reported in Ref. 7. Even though the same trends were observed, the loss of efficiency at low modulation depths was not observed. This may be because lower recording intensities and a longer wavelength were used in Ref. 7. The curve also shows a saturation behavior at $m=\sim 1$. This saturation is not due to the normal diffraction from a strong grating because we have a relatively weak modulation $(2 \pi \Delta n L / \lambda n<0.33)$. Therefore we attribute this saturation to the monomer diffusion mechanism in the film.

One measures the film's response to grating frequency by recording the diffraction efficiency as a function of the angle between the beams while keeping the bisector of the angle perpendicular to the film's surface. This geometry ensures that the fringes are always perpendicular to the film surface.

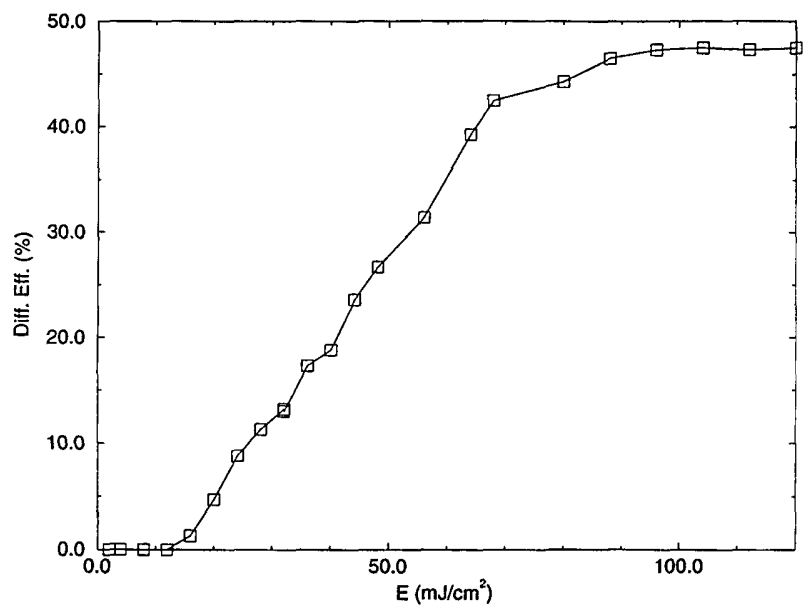

Fig. 2. Diffraction efficiency versus exposure energy.

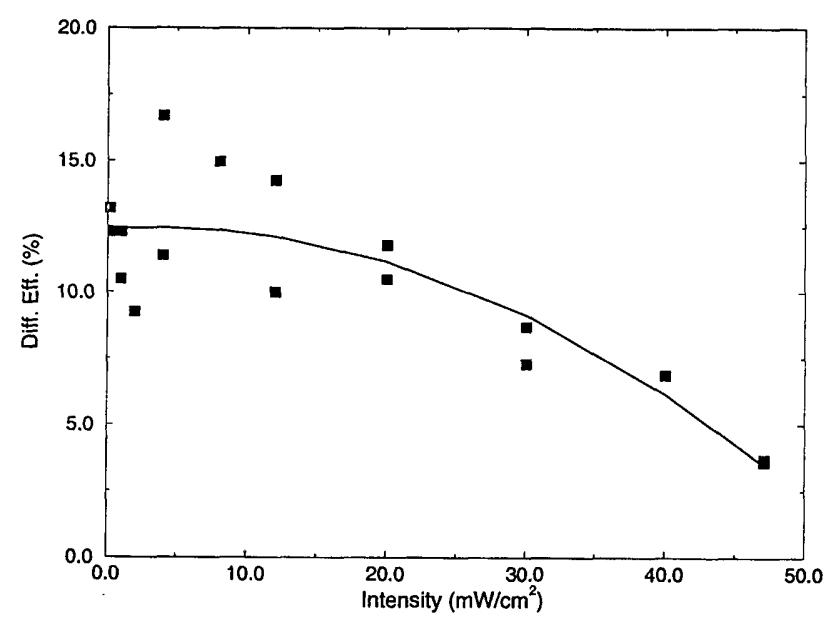

Fig. 3. Diffraction efficiency versus intensity for $E=20 \mathrm{~mJ} / \mathrm{cm}^{2}$.

Figure 5 shows the result for $E=20 \mathrm{~mJ} / \mathrm{cm}^{2}$. The efficiency is plotted versus the full angle between the beams to make it easier to understand the next two results. Notice that the film prefers lower-frequency gratings, but the curve becomes flatter at $\sim 90^{\circ}$ between the beams. The 3 -db point of the frequency response is at $\sim 30^{\circ}$, corresponding to $\sim 1,000$ cycles $/ \mathrm{mm}$.

Figure 6 shows the diffraction efficiency for a total exposure of $20 \mathrm{~mJ} / \mathrm{cm}^{2}$ as a function of reference angle $\theta$ for both polarizations with the signal beam incident normal to the surface. The intensity of each beam is $2.2 \mathrm{~mW} / \mathrm{cm}^{2}$. The results for each polarization are similar. HRF-150 is designed by DuPont as a transmission film, and, as Fig. 5 shows, it does not record reflection holograms effectively. Comparing this result with Fig. 5, we see that the drop in efficiency for angles larger than $30^{\circ}$ is not due entirely to the change in spatial frequency. It appears that the film does not effectively record gratings that have a large tilt angle inside the material. This might be caused by nonuniform shrinkage of the film.

To evaluate this effect further, we again measured the film's response as the reference angle is changed

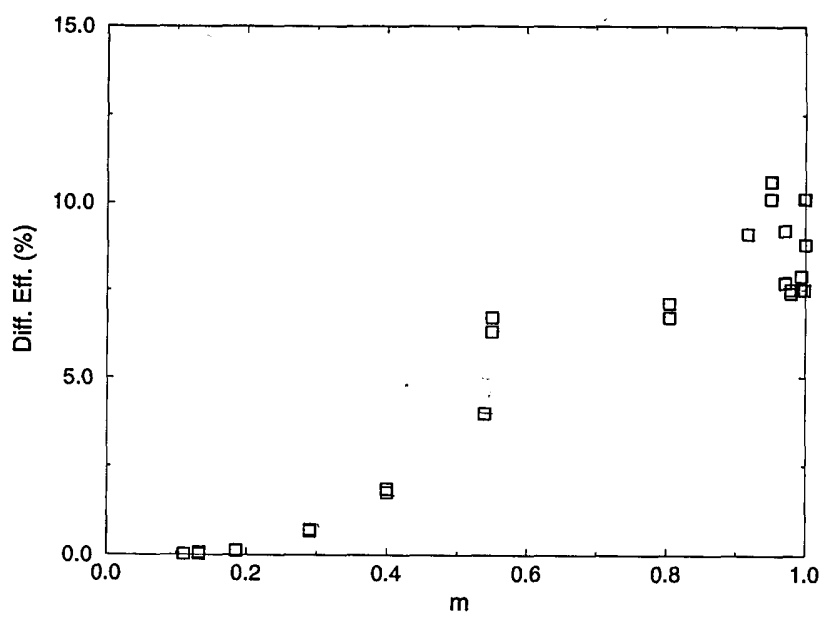

Fig. 4. Diffraction efficiency versus modulation depth. 


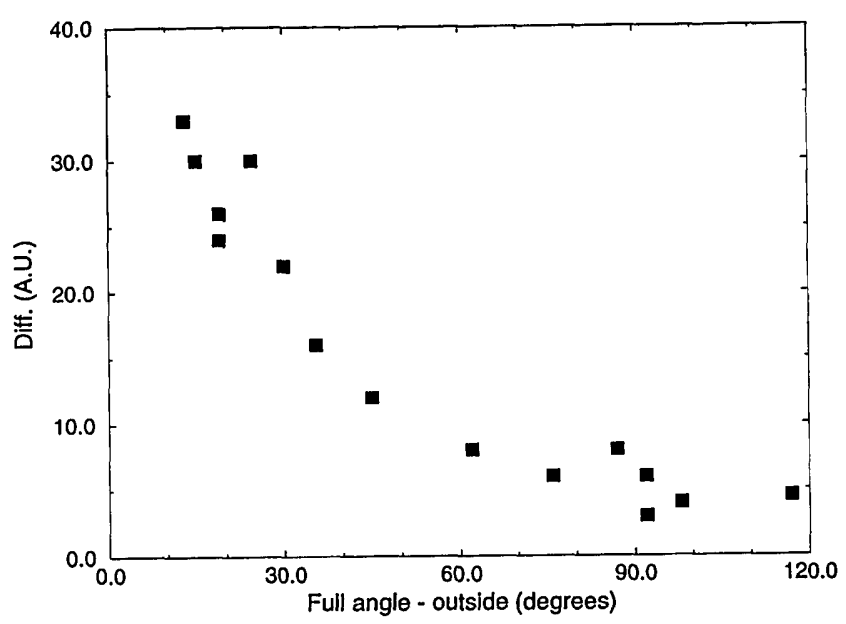

Fig. 5. Diffraction efficiency versus full angle between the beams outside the material for $E=20 \mathrm{~mJ} / \mathrm{cm}^{2}$ and no grating tilt.

but this time with the center angle between the beams being $90^{\circ}$, at which we know (from Fig. 5) that the film's response is relatively flat. Figure 7 plots diffraction efficiency for a total exposure of $20 \mathrm{~mJ} / \mathrm{cm}^{2}$ as a function of reference angle $\theta$ with respect to the film's normal. In this plot an angle of $45^{\circ}$ refers to the $90^{\circ}$ full angle between the beams with no tilt in the gratings. Notice that at $0^{\circ}$ the diffraction is less than at $20^{\circ}$ even though we know that the spatial frequency at $0^{\circ}$ is preferred by the film when the grating is not tilted. This loss in sensitivity is attributed to the grating tilt. The maximum fringe tilt that the film can tolerate is $\sim 10^{\circ}$.

If the material is going to be used as a storage element, the holograms must be able to be recalled nondestructively after they are fixed with UV light. Figure 8 shows the continuous readout of a hologram for $100 \mathrm{~h}$. The reference intensity was $\sim 3 \mathrm{~mW} / \mathrm{cm}^{2}$ with a diffraction efficiency of $\sim 1.5 \%$. The initial increase, shown on the graph, is caused by the bleaching of the material with light exposure. The grating was recorded with the object beam incident at

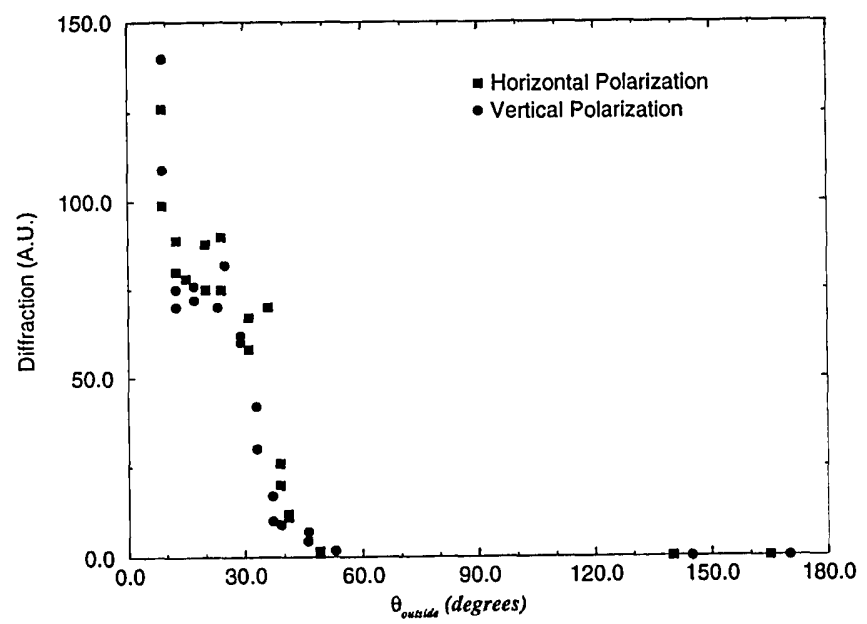

Fig. 6. Diffraction efficiency versus angle outside the material for an $E=20 \mathrm{~mJ} / \mathrm{cm}^{2}$ image beam incident normal to the surface.

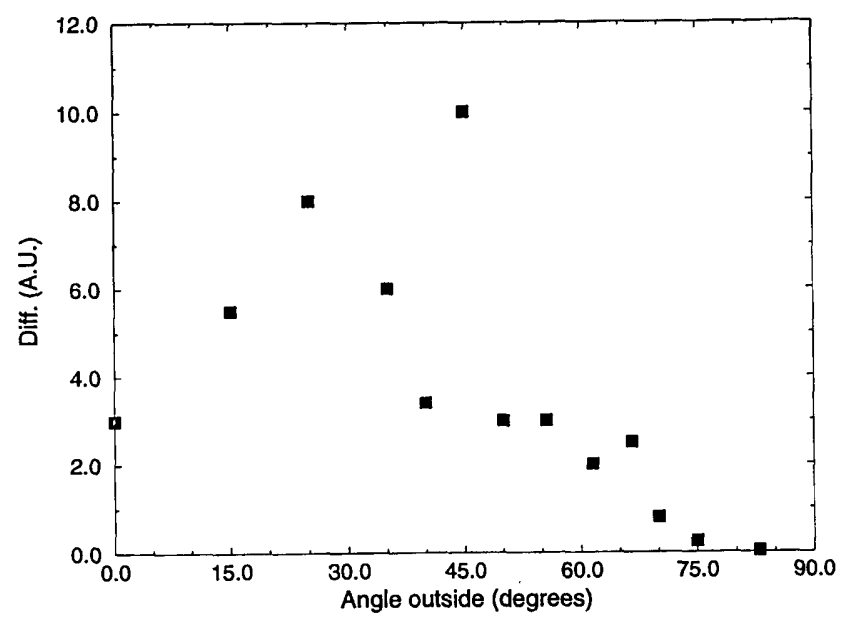

Fig. 7. Diffraction efficiency versus angle outside the material for $E=20 \mathrm{~mJ} / \mathrm{cm}^{2}$ with $90^{\circ}$ between the $0^{\circ}$ reference beam and the signal.

$-45^{\circ}$ and the reference beam at $45^{\circ}$ from the surface normal. Multiplexed image-plane holograms recorded more than a year ago and stored at room temperature could still be recalled.

Whether or not the reaction spreads laterally (in the plane of the film) is important if spatial multiplexing is desired. To test if the reaction spreads, we cut a slit $\sim 1 \mathrm{~mm}$ across and $1 \mathrm{~cm}$ long in tin foil to create a mask. The photopolymer was exposed with a single normal incident beam through this mask $\left(E \approx 300 \mathrm{~mJ} / \mathrm{cm}^{2}\right)$. The sample was then left in the dark, giving the reaction time to polymerize the material in the exposed region and to possibly laterally spread to beyond the illuminated area. The mask was then removed, and a hologram was recorded in a large area around the slit's location on the film. The reconstructed hologram consisted of bright areas in which the film was unaffected by the first exposure and dark areas in which the film had already been exposed. Any lateral spreading of the reaction would cause a gradual loss of efficiency as the reaction spreads out and uses up the film's dynamic range.

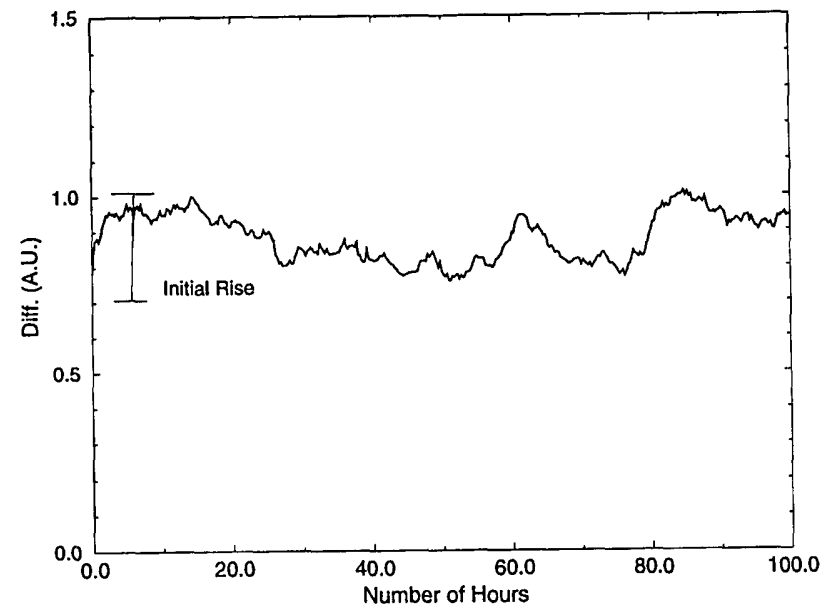

Fig. 8. Diffraction versus readout time demonstrating nondestructive recall. 


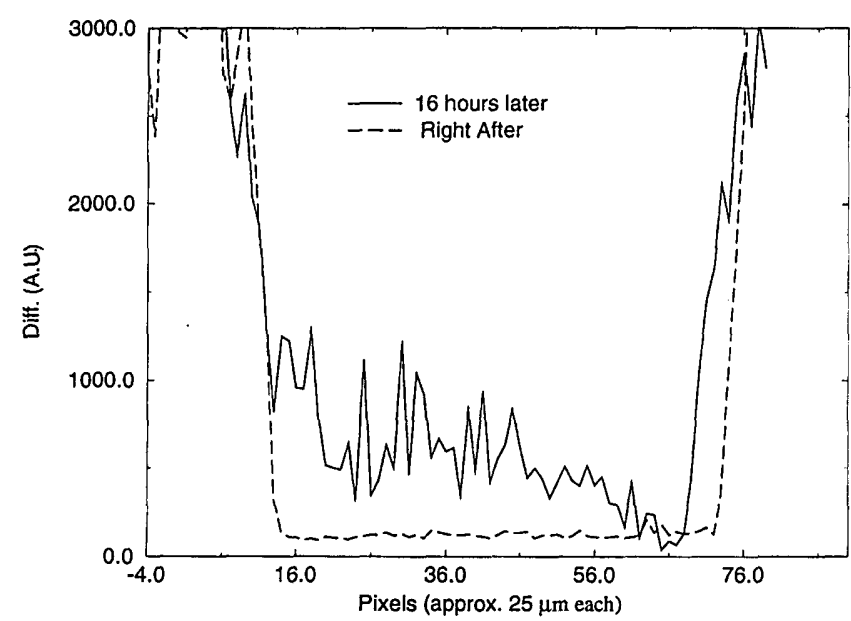

Fig. 9. Cross sections of two reconstructed holograms across pre-exposed areas.

When the second exposure immediately followed the first, no appreciable lateral spread was observed, as shown in Fig. 9. The curve labeled Right After shows a cross section of the intensity of the reconstruction across the dark area. The experiment was repeated, except this time the sample was left in the dark for $16 \mathrm{~h}$ before the holographic exposure was done. Again a dark slit was seen in the hologram, and a cross section labeled as 16 hours later is shown in Fig. 9. Notice that at the transition from dark to light the slope of the two curves is approximately the same. Therefore within a measurement error of $\sim 100 \mu \mathrm{m}$ the reaction does not spread laterally. The 16-hour curve is of slightly narrower width because it was taken at a different place along the slit.

In summary, we have observed that the diffraction efficiency of the HRF-150 photopolymer for a given exposure decreases with increases in intensity and grating tilt angle. The holograms can be nondestruc- tively reconstructed for long periods of time at room temperature. The photoinitiated reaction was seen to spread less than $100 \mu \mathrm{m}$ over periods of many hours. Overall, the HRF-150 has many of the desirable characteristics needed for a practical holographic storage material. The major improvement that is needed is an increase in film thickness of $200 \mu \mathrm{m}$ or more. This would permit more holograms to be multiplexed at a single location, and it would also probably alleviate the problem with tilted gratings that is reported in this paper.

We thank Steve Zager of DuPont and Geoffrey Burr for their helpful discussions. This work was funded at the California Institute of Technology by the U.S. Air Force Office of Scientific Research.

\section{References}

1. D. Psaltis, "Parallel optical memories," Byte 17, 179-182 (1992).

2. K. Curtis and D. Psaltis, "Multi-channel disk-based optical correlator," presented at the Society of Photo-Optical and Instrumentation Engineers Conference on Optical Implementation of Information Processing, San Diego, Calif., 12 July 1993; "Disk-based optical correlator," submitted to Opt. Eng.

3. W. K. Smothers, T. J. Trout, A. M. Weber, and D. J. Mickish, "Hologram recording in DuPont's new photopolymer films," in Practical Holography IV, S. A. Benton, ed., Proc. Soc. PhotoOpt. Instrum. Eng. 1212, 30-39 (1990).

4. K. Curtis and D. Psaltis, "Recording of multiple holograms in photopolymer films," Appl. Opt. 31, 7425-7428 (1992).

5. K. Curtis and D. Psaltis, "Holographic recording in photopolymer films," in OSA Annual Meeting, Vol. 23 of 1992 OSA Technical Digest Series (Optical Society of America, Washington, D.C., 1992), p. 145.

6. K. Curtis, "Phase grating profiles in photopolymers," Opt. Commun. 95, 113-116 (1993).

7. U. Rhee, H. J. Caulfield, J. Shamir, C. S. Vikram, M. M. Mirsalehi, "Characteristics of the DuPont photopolymer for angularly multiplexed page-oriented holographic memories," Opt. Eng. 32, 1839-1847 (1993). 